\title{
Durable Complete Response of a Recurrent Mesencephalic Glioblastoma Treated with Trametinib and Low-Dose Dabrafenib in a Patient with Neurofibromatosis Type 1
}

\author{
Gil Awada $^{a}$ Daphne Serruys ${ }^{b} \quad$ Julia Katharina Schwarze ${ }^{a}$ \\ Lien Van De Voorde ${ }^{c}$ Johnny Duerinck ${ }^{d}$ Bart Neyns ${ }^{a}$ \\ aDepartment of Medical Oncology, Universitair Ziekenhuis Brussel, Brussels, Belgium; \\ ${ }^{b}$ Department of Medical Oncology, AZ Sint-Lucas Gent, Ghent, Belgium; 'Department of \\ Radiation Oncology, AZ Sint-Lucas Gent, Ghent, Belgium; dDepartment of Neurosurgery, \\ Universitair Ziekenhuis Brussel, Brussels, Belgium
}

\author{
Keywords \\ Glioblastoma $\cdot$ Neurofibromatosis type $1 \cdot$ Trametinib $\cdot$ Dabrafenib
}

\begin{abstract}
Patients with neurofibromatosis type 1 (NF1) have an increased lifetime risk for the development of nervous system tumors, including high-grade gliomas (glioblastoma). NF1 is associated with the loss of expression of neurofibromin 1 (NF1 gene product). This hyperactivates the mitogen-activated protein kinase pathway, leading to cellular proliferation and survival. MEK-inhibitor monotherapy is a promising treatment strategy in this setting, but is associated with distinct adverse events, most prominently cutaneous toxicity. We report the case of a young NF1 patient with a recurrent, heavily pretreated mesencephalic glioblastoma who was treated with the MEK-inhibitor trametinib ( $2 \mathrm{mg}$ once daily). A partial response was documented, but unfortunately, he developed dose-limiting cutaneous toxicity (rash, paronychia). Based on interim results of a phase 2 trial in advanced $B R A F^{V 600}$ wild-type melanoma indicating that a low dose of the BRAF-inhibitor dabrafenib is able to counter trametinib-related cutaneous toxicity, dabrafenib $50 \mathrm{mg}$ twice daily was added. The cutaneous adverse events gradually recovered after addition of dabrafenib to trametinib. The patient eventually achieved a durable complete response, has excellent tolerance of his treatment and remains fully active.
\end{abstract}




\section{Introduction}

Neurofibromatosis type 1 (NF1) is an autosomal dominant genetic disease caused by a mutation in NF1, a gene that encodes the tumor suppressor protein neurofibromin 1 . One half of cases are inherited (germline mutation), whilst the other half arise from a de novo mutation [1]. Neurofibromin 1 is expressed in many cell types, including neurons and glial cells, and accelerates the conversion of active GTP-bound RAS to inactive GDP-bound RAS. GTP-bound RAS activates the mitogen-activated protein kinase (MAPK, also called the RAF-MEK-ERKpathway) and AKT-mammalian target of rapamycin (AKT-mTOR) effector pathways, leading to cell growth and survival. Loss of neurofibromin 1 activity leads to hyperactivated RAS which at its turn hyperactivates these effector pathways leading to uncontrolled proliferation $[2,3]$.

Clinical manifestations of NF1 are variable and can be benign or malignant. Some result from monoallelic loss of NF1, while others require biallelic loss of NF1 or other concurrent genetic alterations (such as TP53 mutations).

Patients have a lifetime cancer risk of approximately $60 \%$, with an approximately 40 -fold increased risk of high-grade gliomas [1]. NF1 loss can lead to a spectrum of nervous system malignancies. Neurofibromas (dermal, plexiform, paraspinal) and optic nerve gliomas are more common in childhood and early adolescence, brainstem gliomas during late adolescence, and glioblastoma and malignant peripheral nerve sheath tumors during adult life [1]. First-line treatment of NF1-associated high-grade gliomas does not differ from treatment of sporadic high-grade gliomas.

\section{Case Presentation}

A 19-year-old asymptomatic male patient, who had been diagnosed with NF1 at the age of 14 years in the context of café-au-lait macules, learning difficulty, and obstructive hydrocephalus (germline heterozygous NF1 mutation c.4466A $>$ G in exon 10b), was diagnosed in August 2017 with a mesencephalic gadolinium-enhancing tumoral lesion of $11 \mathrm{~mm}$ in diameter on magnetic resonance imaging of the brain during routine follow-up for hydrocephalus. Due to its location, the lesion was considered unresectable. A stereotactic biopsy revealed a World Health Organization grade IV glioma (glioblastoma). Immunohistochemical staining showed glial fibrillary acidic protein, isocitrate dehydrogenase 1 , transcriptional regulator ATRX, and p53 positivity with a Ki67 positivity of 5-10\%. Fluorescence in situ hybridization did not show an amplification of the epidermal growth factor receptor gene. Further genetic testing of the tumor using next-generation sequencing lead to inconclusive results due to a limited amount of tumor tissue.

The patient was initially treated in September 2017 with radiation therapy (33 fractions of $1.8 \mathrm{~Gy}$, due to the brainstem location) and concomitant temozolomide chemotherapy $\left(75 \mathrm{mg} / \mathrm{m}^{2}\right)$, followed by 2 cycles of adjuvant temozolomide $\left(150-200 \mathrm{mg} / \mathrm{m}^{2}\right)$ after which he progressed in February 2018. He subsequently initiated treatment with the combination of the oral vascular endothelial growth factor receptor 1-3 inhibitor axitinib ( $5 \mathrm{mg}$ twice daily) and the programmed cell death ligand 1 blocking immune checkpoint inhibitor avelumab $(10 \mathrm{mg} / \mathrm{kg}$ ) in a phase 2 clinical trial (NCT03291314). A confirmed complete response was achieved in May 2018. Treatment was continued but unfortunately, progressive disease was documented in December 2018. Third-line treatment with axitinib (5 mg twice daily) plus lomustine $\left(90 \mathrm{mg} / \mathrm{m}^{2}\right)$ was started for a total of 2 cycles, until progression of disease in May 2019. At this point, fourth-line treatment with the MEK-inhibitor trametinib (2 mg once daily) was started (Fig. 1). Within the first month of 


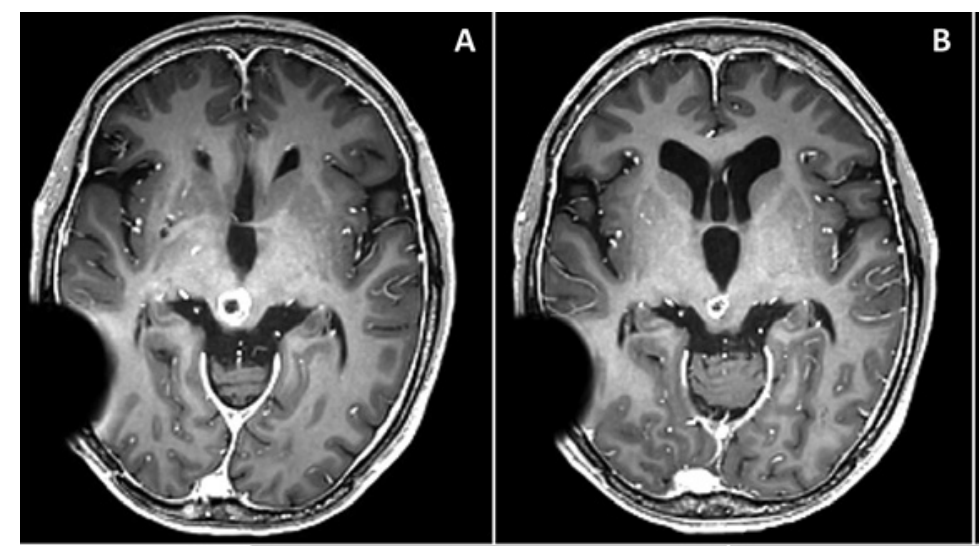

B $C$
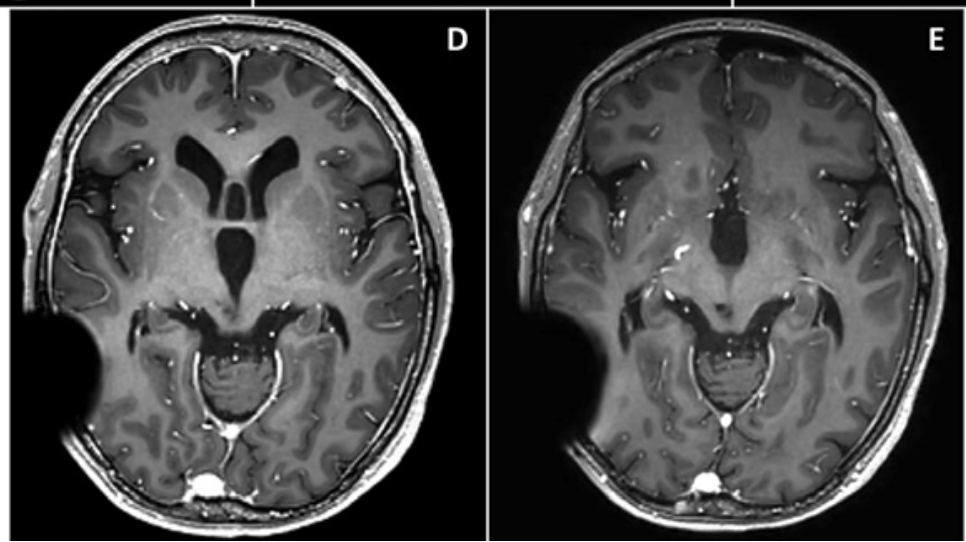

Fig. 1. Axial images from gadolinium-enhanced T1 magnetic resonance imaging of the brain showing the ring-like glioma appearance in the mesencephalon before initiation of trametinib monotherapy (A); partial response 3 months after initiation of trametinib monotherapy (B); confirmed partial response 5 months after initiation of trametinib monotherapy, before the addition of low-dose dabrafenib (C); complete response 4 months after initiating the combination of trametinib and low-dose dabrafenib (D); confirmed complete response 7 months after initiating the combination of trametinib and low-dose dabrafenib (E). The patient has a ventriculoperitoneal shunt for hydrocephalus.

treatment, he developed grade 2 creatine kinase increase and grade 2 acneiform dermatitis on the head and trunk which was treated with topical metronidazole and oral minocycline. Eventually, an interruption of trametinib dosing and subsequent dose reduction to $1.5 \mathrm{mg}$ once daily was required. In August 2019, a partial response was documented (Fig. 1). However, grade 1 acneiform dermatitis persisted, and grade 2 paronychia of the toenails developed which were treated with topical metronidazole, and chloramine and fusidic acid, respectively. Based on interim safety results from an ongoing phase 2 trial indicating that a low dose (50 mg twice daily) of the BRAF-inhibitor dabrafenib counteracts trametinib-related cutaneous toxicity in patients with advanced $B R A F^{V 600}$ wildtype melanoma, dabrafenib $50 \mathrm{mg}$ twice daily was added to trametinib in November 2019 [4]. In March 2020, a complete response was documented. Acneiform dermatitis and the paronychia gradually recovered after the addition of low-dose dabrafenib, and on his latest clinic visit in June 2020, the patient had a confirmed complete response (Fig. 1), excellent tolerance of his treatment and remains fully active. 


\section{Discussion}

Molecular-targeted therapies under investigation for NF1-associated gliomas include mTOR-inhibitors and MAPK-inhibitors, most commonly MEK-inhibitors, that block the hyperactivated AKT-mTOR- and MAPK-pathway, respectively. A phase 2 trial of the oral mTORinhibitor everolimus in NF1-associated pediatric low-grade glioma showed disease control in 15 of 22 patients ( 1 complete and 2 partial responses, 12 stable disease) [5]. A phase 2 trial of the MEK-inhibitor selumetinib in patients with NF1-associated recurrent, refractory, or progressive low-grade glioma showed a confirmed objective response in 10 of 25 evaluable patients $(40 \%)$ and disease control in all 25 patients. The progression-free survival at 2 years was 96\% (95\% confidence interval 74-99\%) [6]. In inoperable plexiform neurofibromas, early phase trials have shown promising activity with trametinib and selumetinib with a confirmed objective response rate of $46-70 \%$. The median duration of response was not reached in either trial $[7,8]$. Clinical benefit has also been reported with trametinib in NF1-associated glioblastoma [9].

Treatment with MEK-inhibitor monotherapy is associated with distinct adverse events, including fatigue, muscular (muscle cramps, creatine kinase increase, rhabdomyolysis), cardiovascular (arterial hypertension, left ventricular ejection fraction decrease), ocular (serous retinopathy, retinal vein occlusion), digestive (nausea, vomiting, diarrhea), and most commonly cutaneous adverse events (acneiform dermatitis, paronychia). Grade 1-2 rash appears in $40-64 \%$ of patients, grade 3-4 rash in 4-12\% [6, 7]. Twenty-five to forty percent of patients treated with MEK-inhibitors require a dose reduction for skin toxicity; permanent treatment interruptions related to cutaneous adverse events are rare but have been reported $[6,7]$.

This report describes the case of a young patient with NF1 who achieved a sustained response of recurrent mesencephalic glioblastoma after treatment with the MEK-inhibitor trametinib. Addition of a low dose of the BRAF-inhibitor dabrafenib increased tolerance of trametinib by reducing trametinib-related cutaneous adverse events without compromising efficacy.

Unfortunately, due to limited tissue availability, loss of function of NF1 or other mutations could not be determined in the tumor lesion. However, genetic studies in patients with NF1-associated high- and low-grade glioma showed that loss of heterozygosity of the germline NF1 mutation was present in the majority of samples [10]. Loss of neurofibromin 1 expression mechanistically leads to a proliferative cellular phenotype through activation of the MAPKpathway. Concurrent BRAF mutations have not been described in patients with NF1-associated glioblastoma and have been described but are rare $(<5 \%)$ in NF1-associated low-grade glioma and malignant peripheral nerve sheath tumors [10-13]. The confirmed response to MEK-inhibition strongly suggests that NF1 loss was the main driver of the MAPK-pathway and, by consequence, cellular survival and proliferation in this patient.

Although dabrafenib is a monomer-selective RAF-inhibitor specifically targeting mutant $\mathrm{BRAF}^{\mathrm{V} 600}$, it also improves tolerance of trametinib by reducing the incidence and severity of skin toxicity. This has been confirmed in large phase 3 trials in advanced $B R A F^{V 600}$ mutant melanoma where the incidence of trametinib-related cutaneous toxicity (acneiform dermatitis, paronychia) is lower with the combination of dabrafenib and trametinib (28\% all-grade) than with trametinib monotherapy (57\% all-grade), at the same trametinib dosing [14, 15]. Adding a low dose (50 mg twice daily) of dabrafenib allows to decrease the incidence of trametinib-related skin toxicity, with a low risk for increasing the incidence of dabrafenibrelated pyrexia, as has been shown by preliminary results of a phase 2 trial in advanced $B R A F^{V 600}$ wild-type (NRAS ${ }^{Q 61 R / K / L}$ mutant or wild-type) melanoma (NCT04059224) [4]. The concept of addition of low-dose dabrafenib to prevent or counter trametinib-related skin 
toxicity is under further investigation in this trial in advanced melanoma but also merits further investigation in NF1-associated glioma and other tumor types driven by MAPKpathway alterations.

\section{Statement of Ethics}

The authors obtained written consent from the patient to publish anonymized information with regard to his case, including imaging results.

\section{Conflict of Interest Statement}

Dr. Awada reports grants and non-financial support from Pfizer; non-financial support from MSD; grants, non-financial support, personal fees from Novartis; non-financial support from Astellas; grants from Kom op tegen Kanker; grants from Stichting tegen Kanker. Dr. Schwarze reports non-financial support from MSD; non-financial support from Amgen. Dr. Neyns reports grants and personal fees from Roche; personal fees from Bristol-Myers Squibb; personal fees from MSD; grants, personal fees and non-financial support from Novartis; personal fees from AstraZeneca; personal fees from CryoStorage; personal fees from EtheRNA; grants from Pfizer; grants from Merck Serono; grants from Stichting tegen Kanker; grants from Kom op tegen Kanker; grants from Fonds voor Wetenschappelijk Onderzoek. All other authors have nothing to declare.

\section{Funding Sources}

None.

\section{Author Contributions}

G.A.: conception, treatment of patient, writing of manuscript. D.S.: treatment of patient, review of manuscript. J.K.S.: treatment of patient, review of manuscript. L.V.D.V.: treatment of patient, review of manuscript.J.D.: treatment of patient, review of manuscript. B.N.: conception, treatment of patient, writing of manuscript.

\section{References}

1 Gutmann DH, Ferner RE, Listernick RH, Korf BR, Wolters PL, Johnson KJ. Neurofibromatosis type 1. Nat Rev Dis Primers. 2017;3:17004.

2 Basu TN, Gutmann DH, Fletcher JA, Glover TW, Collins FS, Downward J. Aberrant regulation of ras proteins in malignant tumour cells from type 1 neurofibromatosis patients. Nature. 1992;356(6371):713-5.

3 Dasgupta B, Yi Y, Chen DY, Weber JD, Gutmann DH. Proteomic analysis reveals hyperactivation of the mammalian target of rapamycin pathway in neurofibromatosis 1-associated human and mouse brain tumors. Cancer Res. 2005;65(7):2755-60.

4 Awada G, Schwarze JK, Reijmen E, Goyvaerts C, Fasolino G, Aspeslagh S, et al. Interim safety and efficacy results of a phase II clinical trial on trametinib and low-dose dabrafenib in patients with advanced BRAFV600 wildtype melanoma. Ann Oncol. 2020;31(Suppl 1):[not available in pubmed,crossref]

5 Ullrich NJ, Prabhu SP, Reddy AT, Fisher MJ, Packer R, Goldman S, et al. A phase II study of continuous oral mTOR inhibitor everolimus for recurrent, radiographic-progressive neurofibromatosis type 1-associated pediatric low-grade glioma: a neurofibromatosis clinical trials consortium study. Neuro Oncol. 2020.

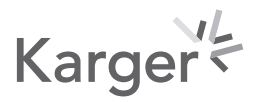


6 Fangusaro J, Onar-Thomas A, Young Poussaint T, Wu S, Ligon AH, Lindeman N, et al. Selumetinib in paediatric patients with BRAF-aberrant or neurofibromatosis type 1-associated recurrent, refractory, or progressive low-grade glioma: a multicentre, phase 2 trial. Lancet Oncol. 2019;20(7):1011-22.

7 Gross AM, Wolters PL, Dombi E, Baldwin A, Whitcomb P, Fisher MJ, et al. Selumetinib for children with plexiform neurofibromas. Lancet Oncol. 2020;18(2):e69-42.

8 McCowage GB, Mueller S, Pratilas CA, Hargrave DR, Moertel CL, Whitlock J, et al. Trametinib in pediatric patients with neurofibromatosis type 1 (NF-1)-associated plexiform neurofibroma: a phase I/IIa study. J Clin Oncol. 2018;36(15_Suppl):10504.

9 Ameratunga M, McArthur G, Gan H, Cher L. Prolonged disease control with MEK inhibitor in neurofibromatosis type I-associated glioblastoma. J Clin Pharm Ther. 2016;41(3):357-9.

10 D'Angelo F, Ceccarelli M, Tala, Garofano L, Zhang J, Frattini V, et al. The molecular landscape of glioma in patients with neurofibromatosis 1. Nat Med. 2019;25(1):176-87.

11 Kaplan HG, Rostad S, Ross JS, Ali SM, Millis SZ. Genomic profiling in patients with malignant peripheral nerve sheath tumors reveals multiple pathways with targetable mutations. J Natl Compr Canc Netw. 2018;16(8): 967-74.

12 Hirbe AC, Pekmezci M, Dahiya S, Apicelli AJ, Van Tine BA, Perry A, et al. BRAFV600E mutation in sporadic and neurofibromatosis type 1-related malignant peripheral nerve sheath tumors. Neuro-oncology. 2014;16(3): 466-7.

13 Shibahara I, Sonoda Y, Suzuki H, Mayama A, Kanamori M, Saito R, et al. Glioblastoma in neurofibromatosis 1 patients without IDH1, BRAF V600E, and TERT promoter mutations. Brain Tumor Pathol. 2018;35:10-8.

14 Flaherty KT, Robert C, Hersey P, Nathan P, Garbe C, Milhem M, et al. Improved survival with MEK inhibition in BRAF-mutated melanoma. N Engl J Med. 2012;367(2):107-14.

15 Robert C, Grob JJ, Stroyakovskiy D, Karaszewska B, Hauschild A, Levchenko E, et al. Five-year outcomes with dabrafenib plus trametinib in metastatic melanoma. N Engl J Med. 2019;381(7):626-36. 